\title{
NOUVELLE
}

\section{La galectine-1 est un ligand du récepteur des lymphocytes pré-B}

Laurent Gauthier, Benjamin Rossi, Claudine Schiff dont certaines permettent la différenciation des précurseurs $B$ en lymphocytes matures pré-B. En utilisant la SLC humaine recombinante comme son-
Centre d'Immunologie Inserm-Cnrs de Marseille-Luminy, case 906 ,

13288 Marseille Cedex 9, France.

schiff@ciml.univ-mrs.fr de, nous avons isolé par biochimie préparative, et identifié par spectrométrie de masse, une molécule exprimée par les cellules stromales qui se lie au préBCR: la galectine-1 (GALI).

GALl est une S-lectine qui récepteur fonctionnel, le pré-BCR (Figure IB). L'étape d'association de la SLC avec la chaîne $\mu$ néosynthétisée constitue un point de contrôle essentiel de la constitution du pré-BCR et du développement B. En effet, chez l'homme et chez la souris, le pré-BCR est impliqué dans l'amplification de la population pré- $B$, dans la sélection du répertoire des chaînes $\mu$, et il influence positivement l'expression transcriptionnelle des gènes des chaînes légères $\kappa$ [1]. Le mécanisme physiologique par lequel le pré-BCR est activé in vivo était jusqu'à présent inconnu. Notre laboratoire vient de démontrer l'existence d'un ligand pour le récepteur pré-B qui est produit par l'environnement médullaire [2].

Les cellules «stromales » constituant l'environnement médullaire participent à la différenciation $B$ en établissant des contacts directs avec les précurseurs $B$ par I'intermédiaire de molécules d'adhérence comme VCAM-1 (vascular cell adhesion molécule) dont le récepteur sur le précurseur B est l'intégrine VLA-4 (ou $\alpha 4 \beta 1$, ou CD49dCD29) et en sécrétant des facteurs solubles comme l'interleukine-7, le SCF (stem cell factor), Flt3-L ou la chimiokine SDF-1 (stromal-derived factor 1, le ligand de (XRCR4), tous susceptibles de contrôler la croissance, la maturation et la survie des précurseurs B (Figure 1C). Or, nous avons démontré récemment que la SLC et des molécules pré-BCR humaines, produites sous forme soluble, étaient capables de se lier de façon spécifique à certaines de ces cellules stromales adhérentes, issues de plusieurs espèces et de plusieurs tissus, et appartient à la famille des galectines, très conservée au cours de l'évolution. Les galectines possèdent une spécificité de liaison pour les $\beta$-galactosides et leurs domaines de reconnaissance des sucres sont très homologues. Nous avons établi que la SLC et GALl interagissent de façon directe, avec une constante d'affinité de $2.10^{6} \mathrm{M}^{-1}$. Cette interaction ne fait pas intervenir les sucres et implique un site de liaison sur GALl différent de la poche se liant aux sucres. GALl est une molécule soluble, son ancrage à la surface des cellules stromales se fait par l'intermédiaire de contre-récepteurs glycosylés, et la fixation de la SLC aux cellules stromales est dépendante de la présence de GALl ancrée à la membrane cellulaire. Lorsqu'on examine par microscopie confocale la localisation du pré-BCR et de GALl lors de l'interaction entre les cellules pré-B et des lignées stromales, on trouve une co-localisation des deux molécules au niveau de la zone de contact entre les deux cellules, entraînant la formation d'une synapse (Figure 1D). Comme le montre la Figure 1, la localisation du pré-BCR dans la synapse est toujours incluse dans celle de GALl, suggérant que GALl, outre sa liaison au pré-BCR, peut aussi interagir avec des contre-récepteurs présents à la surface des cellules pré-B. Enfin, nos résultats montrent que la formation de la synapse entre les cellules pré-B et stromale s'accompagne de la mise en route d'une activité intracellulaire de phosphorylation des tyrosines et d'un signal de transduction à 
partir du pré-BCR. Un modèle visualisant l'organisation moléculaire de la synapse, qui peut être qualifiée de « développementale », est présenté sur la Figure $1 \varepsilon$.

La nature des contre-récepteurs de la galectine-l qui sont impliqués dans l'établissement de la synapse entre les cellules
pré-B et stromales reste à déterminer. Néanmoins, des contre-récepteurs de la galectine-1 ont déjà été identifiés dans d'autres systèmes biologiques: il s'agit de protéines de la matrice extracellulaire (laminine et fibronectine) ou de récepteurs de surface comme CD45, CD43, CD7,

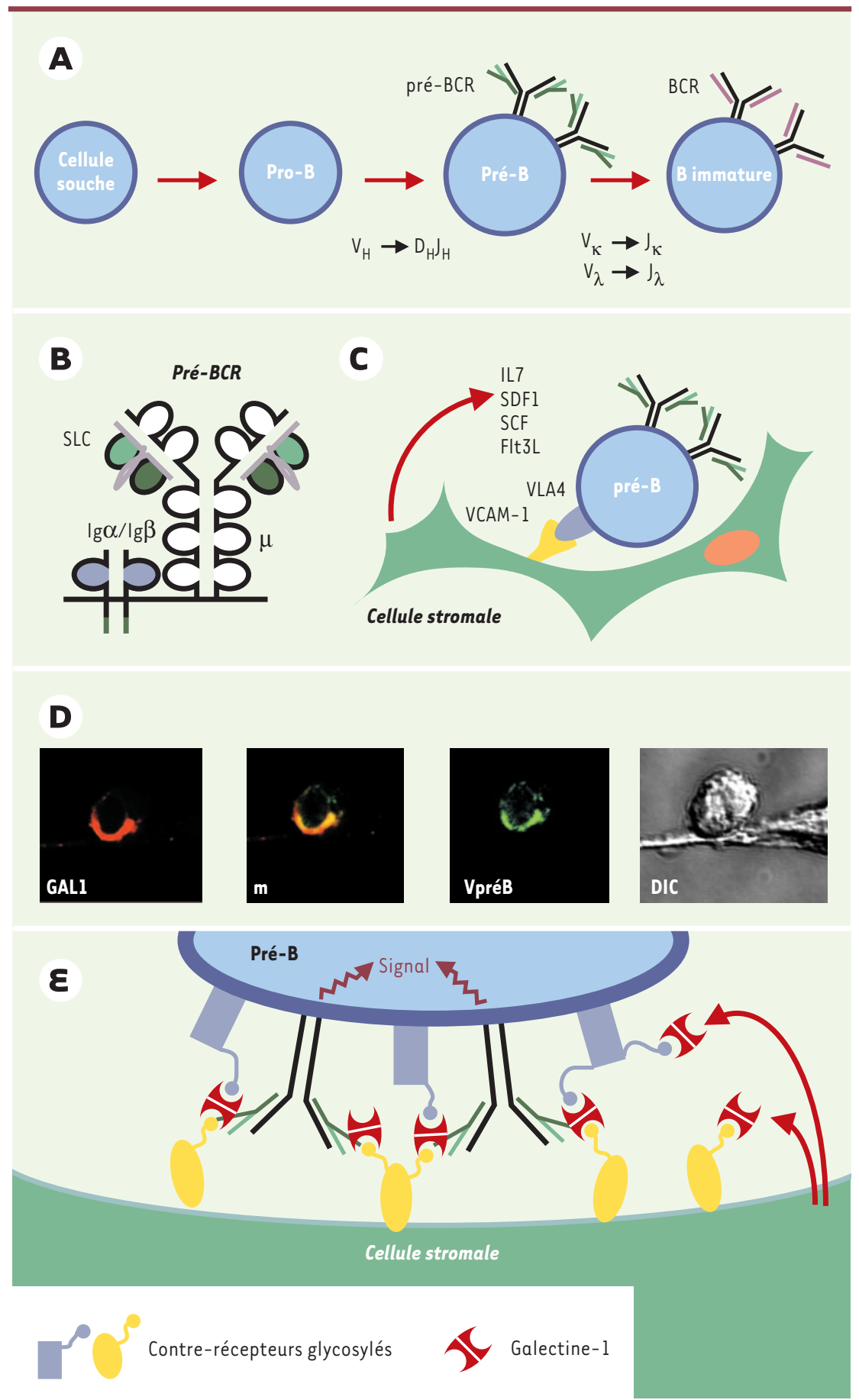

Figure 1. Expression et rôle du pré-BCR et de son ligand la galectine-1 au cours de la différenciation lymphoïde B. A. Les différentes étapes de la différenciation des lymphocytes $B$ ont lieu dans la moelle osseuse chez l'adulte et sont caractérisées par le réarrangement séquentiel des gènes des immunoglobulines. Les réarrangements commencent au niveau des gènes codant pour la chaîne lourde $\mathrm{H}\left(\mathrm{D}_{\mathrm{H}^{-}} \mathrm{J}_{\mathrm{H}}\right.$ suivi de $\mathrm{V}_{\mathrm{H}^{-}} \mathrm{D}_{\mathrm{H}^{-}}$ $J_{H}$ ) puis au niveau des locus codant pour la chaîne légère $L, \kappa\left(V_{\kappa}-J_{\kappa}\right)$ puis $\lambda\left(V_{\lambda}-J_{\lambda}\right)$. Les cellules pré- $B$ et $B$ immatures expriment respectivement les récepteurs pré- $B$ (pré- $B C R$, en noir) et $B(B C R)$. $B$. Le pré-BCR est formé de la surrogate light chain (SLC, en vert), composée des protéines $\lambda$-like et Vpré-B et de la chaîne lourde $\mu$ (en noir), associée aux molécules de transduction $\lg \alpha / \lg \beta$ codées par les gènes CD79a et CD79b. C. Les cellules stromales de la moelle osseuse sont indispensables à la croissance, la maturation et la survie des précurseurs B. Elles établissent des contacts étroits avec les progéniteurs lymphoïdes par l'intermédiaire de molécules d'adhérence (VCAM/VLA4) et sécrètent des facteurs solubles (IL-7, SCF, Flt3L, SDF-1) qui sont délivrés aux précurseurs. D. Lorsque les cellules pré-B sont cultivées sur des lignées stromales, nous avons observé par microscopie confocale que le pré-BCR (révélé par un anticorps anti-Vpré-B, en vert) et la galectine-1 (révélé par un anticorps anti-GALl, en rouge) sont tous deux re-localisés à la zone de contact entre les deux cellules participant à la formation d'une synapse. De plus, cette relocalisation s'accompagne de l'induction d'un signal de transduction à partir du pré-BCR. $\varepsilon$. Nous proposons un modèle de l'organisation moléculaire de la synapse « développementale » qui s'établit entre la cellule pré-B et la cellule stromale. La galectine-1, sécrétée par la cellule stromale, est capturée par des contrerécepteurs présents à la surface des deux cel-

lules ainsi que par les pré-BCR. La galectine-l se comporte comme l'organisateur moléculaire d'un maillage qui rassemble les contre-récepteurs et les récepteurs pré-B. La formation de ce réseau est responsable de la re-localisation et du déclenchement de la signalisation des pré-BCR. 
par les différents contre-récepteurs qui déterminent la nature des réponses biologiques [4]. Les galectines induisent des effets contrastés sur la croissance cellulaire, et l'effet biologique observé - prolifération ou apoptose - dépend du type cellulaire et du statut d'activation cellulaire. Par exemple, il a été rapporté que GALl pouvait à la fois jouer un rôle d'inhibition de la prolifération sur les cellules $\mathrm{T}$, et promouvoir la prolifération des cellules endothéliales vasculaires. Les galectines jouent aussi un rôle crucial dans les processus de transformation cellulaire et dans la formation des métastases.

II nous reste à déterminer quel est le rôle physiologique de GALl sur les cellules préB. La propriété remarquable qu'a cette lectine d'organiser - à l'interface entre le précurseur B et la cellule stromale - un réseau de contre-récepteurs glycosylés dans lequel le pré-BCR est engagé et stimulé laisse présager un rôle fonctionnel déterminant d'une telle organisation in vivo. Compte tenu de la perte de la population pré-B chez les souris $S L C^{-/-}$[5], la formation de la synapse entre les cellules pré-B et stromales pourrait être essentielle pour stimuler l'entrée en cycle des cellules pré-B et pour assurer la transition entre les grandes et les petites cellules pré-B, qui caractérise la différenciation lymphocytaire. $\diamond$

Galectin- 1 is a ligand

for the pre-B cell receptor

\section{RÉFÉRENCES}

1. Melchers F, ten Boekel $\varepsilon$, Seidl T, et al. Repertoire selection by pre-B-cell receptors and $B$-cell receptors, and genetic control of B-cell development from immature to mature $B$ cells. Immunol Rev 2000; 175: 33-46.

2. Gauthier L, Rossi B, Roux F,
Termine $\varepsilon$, Schiff C. Galectin-1 is a stromal cell ligand of the pre-BCR implicated in synapse formation between pre-B and stromal cells and in pre-BCR triggering. Proc Natl Acad Sci USA 2002; 99: 13014-9.

3. Rabinovich GA, Baum LG, Tinari N, et al. Galectins and their ligands: amplifiers, silencers or tuners of the inflammatory response? Trends Immunol 2002; 23: 313-20.

4. Hughes RC. Galectins as modulators of cell adhesion. Biochimie 2001; 83: 667-76.

5. Shimizu T, Mundt C, Licence $\mathrm{S}$, Melchers F, Martensson IL. VpreB1/VpreB2/lambda 5 triple-deficient mice show impaired B cell development but functional allelic exclusion of the IgH locus. J Immunol 2002; 168: 6286-93.

\section{NOUVELLE}

\section{La levure, une aide pour décrypter les maladies mitochondriales humaines?}

Françoise Foury

> La séquence complète d'un génome eucaryote fut obtenue pour la première fois en 1996 chez la levure de boulanger Saccharomyces cerevisiae [1]. Il apparut alors que la moitié des gènes avaient échappé aux cribles de la mutagenèse classique et que, pour bon nombre d'entre eux, leur fonction ne pouvait être prédite par l'analyse de leur séquence. Des études d'analyse fonctionnelle furent alors développées chez $S$. cerevisiae, comme le projet EUROFAN, financé par la Commission européenne, qui conduisit au clonage et à la délétion individuelle de 700 gènes de $S$. cerevisiae de fonction totalement inconnue (http://mips.gsf.de/proj/eurofan/index. html). Récemment, un consortium américano-européen a construit 5916 mutants délétés, soit une collection presque complète du génome $(96,5 \%$ des phases ouvertes de lecture annotées [ORF, open reading frame] de S. cerevisiae) [2-3]. Chaque gène a été remplacé par une cassette de délétion contenant le gène bactérien $K_{a n}{ }^{R}$ responsable chez la levure de la résistance à la généticine [4], et de part et d'autre de celui-ci, deux séquences de 20 nucléotides distinctes et différentes pour chaque délétion (Figure 1). Ces séquences, qui constituent des code-barres caractérisant spécifiquement chaque gène délété, permettent d'analyser en parallèle un
Unité de Biochimie

physiologique,

Université Catholique

de Louvain,

place Croix du Sud, 2-20,

1348 Louvain-la-Neuve,

Belgique

foury@fysa.ucl.ac.be

mélange de souches délétées. La collection de souches délétées est cultivée dans un milieu donné et des échantillons cellulaires sont prélevés au cours de la croissance. L'ADN des cassettes de délétion est extrait et amplifié par PCR grâce à deux oligonucléotides communs à toutes les cassettes de délétion, puis il est hybridé à des puces à ADN Affymétrix ${ }^{\circledR}$ correspondant aux 11832 code-barres des cassettes de délétion. Dans un mélange de souches, l'abon- 